14.

\section{Health Related Quality of Life Among} Survivors of Prehospital Cardiac Arrest

Vipin Jain, MBBS, *Jennifer A. Brancel, BS, David A. Bullard, BA, Michael R Sayre, MD, ToddF. Breaux, BS, MichaelF. Uphus, EMT-1 Department of Emergency Medicine, University of Cincinnati, Cincinnati, Ohio USA

Purpose: The purpose of this study was to measure the quality of life of cardiac arrest long-term survivors.

Design: Prospective outcome evaluation.

Setting: The emergency medical system of a mid-sized city.

Type of participants: Prehospital cardiac arrest victims who had a resuscitation attempt from 5/93 through 12/95 and were discharged alive. Patients $<18$ were excluded.

Measurements: Patients were surveyed by mail. Non-responders were telephoned. The questions assessed Glasgow-Pittsburgh overall performance (GPOP) scores before and after the cardiac arrest. Additional items assessed physical, role, affective, and cognitive functioning, and global health status.

Main Results: 760 cases were collected. 40 had unknown vital status at hospital discharge. The survival rate to hospital discharge for the 270 patients with cardiac etiology and initial rhythm of ventricular fibrillation was $15 \%$, and it was $6 \%$ for the other 450 patients. Of 68 survivors, 23 died before a survey was sent; two had unknown addresses. 21 patients (49\%) responded, and four refused to participate. The mean interval from cardiac arrest to survey response was 531 days. Ten reported good post-arrest GPOP scores, 5 had moderate disability, 4 severe disability, and 2 were comatose. Cognitive function was improved in 1, unchanged 6 , slightly worse 7 , markedly worse 4 , and none in 3 . Eleven patients reported feeling happy. Global health was excellent for 2 , very good 8 , good 5 , fair 2, and poor 3 .

Conclusion: About half of long-term survivors report good or excellent functioning after cardiac arrest. More than 2/3rds are in good health, and most are happy.
19.

\section{A Breathing Manikin Model for Teaching Nasotracheal Intubation to EMS Professionals}

Juan March, $M D,{ }^{*}$ Kathleen Dunn, MD, MSPA, Lawrence Brown, EMT-P, Johnny Farrow, $M D$, Phillip Perkins, $M D$

Department of Emergency Medicine, East Carolina University School of Medicine, Greenville, North Carolina USA

Purpose: The wide spread use of orotracheal intubation with rapid sequence induction has made it difficult for EMS professionals to gain experience in nasotracheal intubation (NTI) in a controlled supervised setting. The purpose of this study was to determine if a training session on NTI with a breathing manikin can be used to improve skill and comfort of EMS professionals.

Methods: A prospective trial was conducted with a convenience sample of 16 emergency medical service professionals, previously trained in nasotracheal intubation techniques. For the training session a Laerdal airway manikin was modified by replacing the lungs with a bag-valve mask device, to simulate breathing with an inspiratory and expiratory phase. Following verbal instruction, and with direct supervision, each participant practiced NTI using the breathing manikin. Each participant completed a questionnaire, both before and after the training session, to determine self assessed comfort and skill level for both oral and nasal intubations $(0=$ lowest, $10=$ highest $)$. The pre and post intervention scores were compared using the Wilcoxon signed-rank test, $£=0.01$.

Results: Following the training session, the comfort level for NTI by participants increased significantly from 2.7 to 6.8 ( $p=$ 0.0001 ). Furthermore, the self assessed skill level for NTI following the training session increased significantly from 2.7 to $6.9(p=0.0001)$. As expected there were no significant differences noted for comfort or skill level for orotracheal intubation following the training session.

Conclusion: For emergency medical service professionals, a training session for nasotracheal intubation using a relatively inexpensive and easily assembled breathing manikin model increases both self assessed comfort and skill level. 